\title{
Possibility of Turbulent Crystals
}

\author{
V.I. Yukalov ${ }^{1}$ and E.P. Yukalova ${ }^{2}$ \\ ${ }^{1}$ Bogolubov Laboratory of Theoretical Physics \\ Joint Institute for Nuclear Research \\ Dubna 141980, Russia \\ ${ }^{2}$ Laboratory of Informational Technologies \\ Joint Institute for Nuclear Research \\ Dubna 141980, Russia
}

\begin{abstract}
The possibility for the occurrence in crystals of a phenomenon, resembling turbulence, is discussed. This phenomenon, called heterophase turbulence, is manifested by the fluctuational appearance inside a crystalline sample of disordered regions randomly distributed in space. The averaged picture for such a turbulent solid is exemplified by an exactly solvable lattice-gas model. The origin of heterophase turbulence is connected with stochastic instability of quasi-isolated systems.
\end{abstract}




\section{Typical Stages of Motion}

The term turbulence is usually applied to characterizing chaotic motion of fluids [1]. Therefore, it could sound strange to mention turbulence in connection with solids. Nevertheless, a similar phenomenon can occur in the latter, being related to mesoscopic heterophase fluctuations [2].

We shall keep in mind everywhere below the word turbulence in its standard sense [3] as characterizing the motion possessing the following main stochastic properties: (i) sensitive dependence on initial conditions, due to exponential divergence of initially close trajectories; (ii) chaotic space structure at fixed moments of time; (iii) existence of a strange attractor. Note that Ruelle [4] used the title of turbulent crystal with regard to a hypothetical stable amorphous solid. However, real amorphous solids are always only metastable.

Turbulence is a spatio-temporal phenomenon. In order to describe spatial and temporal scales typical of heterophase turbulence, let us recall what are, in general, characteristic scales for a system of interacting particles.

The first evident characteristic length is an effective interaction radius $r_{i n t}$ or a scattering length $a_{s} \sim r_{\text {int }}$. If a characteristic particle velocity is $v$, then the interaction time is

$$
\tau_{i n t} \sim \frac{r_{i n t}}{v} .
$$

In the temporal interval $0<t<\tau_{\text {int }}$, called the dynamic stage, particles move, to a first glance, randomly.

Another important length is a mean free path $\lambda_{m f p}$ defining the local-equilibrium time

$$
\tau_{l o c} \sim \frac{\lambda_{m f p}}{v} .
$$

And the temporal diapason $\tau_{\text {int }}<t<\tau_{\text {loc }}$ is the kinetic stage. At this stage, because of mutual interactions, interparticle correlations begin arising. For the density of particles $\rho \sim a^{-3}$, with $a$ being a mean interparticle distance, the mean free path is $\lambda \sim\left(\rho a_{s}^{2}\right)^{-1}$, that is, $\lambda \sim a^{3} / a_{s}^{2}$. Therefore,

$$
\tau_{l o c} \sim\left(\frac{a}{r_{\text {int }}}\right)^{3} \tau_{\text {int }} .
$$

In condensed matter, $a \sim r_{i n t}$, because of which $\tau_{l o c} \sim \tau_{\text {int }}$, and the kinetic stage being practically absent.

After the local equilibrium time $\tau_{l o c}$, the state of local equilibrium is being evolved, and for $t>\tau_{\text {loc }}$, one has the hydrodynamic stage. But the latter consists of two qualitatively different parts. There exists a coherence radius $r_{\text {coh }}$ characterizing the effective size of mesoscopic heterophase fluctuations. The related coherence time is

$$
\tau_{c o h} \sim \frac{r_{c o h}}{v} .
$$

Heterophase fluctuations are mesoscopic because the coherence radius is between the mean interparticle distance $a$ and the linear size of the whole system $L$,

$$
a \ll r_{c o h} \ll L .
$$


The coherence time $\tau_{c o h} \gg \tau_{l o c}$ defines an average lifetime of a mesoscopic fluctuation. The temporal interval $\tau_{l o c}<t<\tau_{c o h}$ corresponds to the mesoscopic stage, when heterophase fluctuations look as frozen, making the system nonuniform.

For times $t>\tau_{c o h}$, heterophase fluctuations become dynamic. They arise randomly in time, being chaotically located in space. Their appearance signifies the existence of local instabilities leading to divergence of initially close trajectories. At each fixed moment of time, the spatial structure of the system is chaotic. Dynamics of a system with heterophase fluctuations corresponds to the evolution on a strange attractor [2]. These characteristics are the main features of a turbulent motion [3]. This is why the occurrence of heterophase fluctuations can be called heterophase turbulence. This phenomenon can be noticed only on the macroscopic stage during times $t>\tau_{c o h}$.

Heterophase fluctuations are rather ubiquitous in nature [2]. They can be clearly observed by various experimental techniques. For instance, in many magnetic alloys, they have been studied by neutron scattering [5]. Mesoscopic heterophase fluctuations are common in liquid crystals [6], high-temperature superconductors [7-10], and many other materials [2]. Anomalous atomic diffusion in solids [11] and anomalous transport in some dynamic systems [12] can be explained by mesoscopic fluctuations. Such an anomalous diffusion is an analog of the turbulent diffusion [3]. Highly unusual properties of colossal magnetoresistive manganites are due to the coexisting fluctuating domains of semiconducting nonmagnetic states and metallic ferromagnetic states [13]. A good example of such a colossal magnetoresistive manganite is $\mathrm{La}_{1-x} \mathrm{Ca}_{x} \mathrm{M}_{n} \mathrm{O}_{3}$, with $x \approx 0.33$, where the fraction of the material in each of two well-defined states strongly depends on temperature and magnetic field, the inhomogeneity being characterized by a mesoscopic scale of about $100 \stackrel{\AA}{A}$. Dynamical JahnTeller distortions around the antiferromagnetic to paramagnetic phase transition in $\mathrm{UO}_{2}$ [14] and $\mathrm{N}_{p} \mathrm{O}_{2}$ [15] are also a kind of mesoscopic fluctuations. Such local fluctuations arise in many liquids, including water, as mesoscopic cooperative structures of different polymorphism [2,16-20], making a system dynamically heterogeneous. Seismic precursors related to local polymorphic transformations [21] can be viewed as mesoscopic heterophase phenomena. This concerns as well the appearance of droplets of quark-gluon plasma in a bulk hadronic medium and of hadronic bubbles in a bulk quark-gluon plasma, occurring around the quark-hadron transition $[22,23]$. Let us stress that heterophase fluctuations should not be confused with formally introduced microscopic clusters in the so-called equilibrium cluster approximations [24,25], but these fluctuations represent nonequilibrium mesoscopic germs with properties qualitatively differing from the surrounding matter.

\section{Averaged Picture of Turbulence}

There exists a statistical approach to describing turbulent motion in terms of an averaged picture [1,3]. Similarly to this, it is possible to develop an averaged picture of heterophase turbulence by averaging over heterophase fluctuations [2].

The appearance of turbulence in fluids happens when the Reynolds number surpasses a critical value. Analogously, heterophase turbulence arises only under a specific combination of interaction parameters and external variables, such as temperature or pressure. The Reynolds number for fluids is the ratio $v L / \nu$, in which $v$ is a characteristic velocity of motion, $L$ is a characteristic linear size, and $\nu$ is kinematic viscosity. That is, the Reynolds number is 
the ratio of factors favoring turbulence to those stabilizing laminar motion. A counterpart of the Reynolds number for heterophase turbulence could be the ratio of interaction parameters favouring a disordered state to those stabilizing an ordered state. Although the definition of such a typical ratio for heterophase turbulence is not always simple and, in addition, depends on thermodynamic variables.

In crystals, heterophase turbulence is manifested by the emergence of chaotically distributed regions of crystalline disorder [16,17]. Factors favoring the appearance of such regions of disorder could be a high concentration of defects and strong anharmonicity. Good candidates for this could be quantum crystals [26] and strongly anharmonic crystals [27,28]. The realistic description of such crystals involves rather complicated techniques. In order to avoid mathematical complications, we shall resort here to a simple lattice-gas model [29] serving as a cartoon of a crystal with many defects or of a solid with pores and cracks [30]. An advantage of employing this model is the possibility to suggest its rigorous analysis and to explicitly demonstrate that heterophase turbulence can correspond to a more stable state than a uniform homophase state.

Consider a system of $N$ particles in volume $V$. Particles are distributed on a crystalline lattice so that each lattice site can be occupied by not more than one particle. The number of lattice sites $N_{s}$ can be larger than that of particles, $N_{s} \gg N$; thus vacant sites can be present. Assume that the distribution of vacancies is not uniform over the system but that vacancies fluctuationally group in some parts of the sample, as a result of which the system possesses essentially different density of particles in different spatial regions, the latter being distributed chaotically. Such a picture is typical of heterophase turbulence, when a more dense phase and more dilute phase randomly fluctuate in space and time.

In an averaged description, the total number of particles can be presented as the sum $N=N_{1}+N_{2}$ of particles pertaining to the dense phase, $N_{1}$, and of those in the dilute phase, $N_{2}$. The whole volume of the system consists of two parts, $V=V_{1}+V_{2}$, those occupied by the dense and dilute phases, respectively. Each volume $V_{\nu}$, with $\nu=1,2$, does not form a connected part filled by the corresponding phase, but is composed of a large number of disconnected mesoscopic regions randomly located in space.

Since the number of sites, $N_{s}$, is larger than the total number of particles, $N$, the density of sites is higher than that of particles,

$$
\rho_{s}>\rho, \quad \rho_{s} \equiv \frac{N_{s}}{V}, \quad \rho \equiv \frac{N}{V} .
$$

By definition, the dense phase is characterized by a higher density of particles that the dilute phase,

$$
\rho_{1}>\rho_{2}, \quad \rho_{\nu} \equiv \frac{N_{\nu}}{V_{\nu}} \quad(\nu=1,2) .
$$

The geometric probabilities of the corresponding phases are

$$
w_{\nu} \equiv \frac{V_{\nu}}{V} \quad\left(w_{1}+w_{2}=1,0 \leq w_{\nu} \leq 1\right) .
$$

The average density of particles can be written as

$$
\rho=w_{1} \rho_{1}+w_{2} \rho_{2} .
$$


It is convenient to introduce the dimensionless densities

$$
n_{\nu} \equiv \frac{\rho_{\nu}}{\rho_{s}} \quad n \equiv \frac{\rho}{\rho_{s}} .
$$

Then Eq. (4) reads

$$
n=w_{1} n_{1}+w_{2} n_{2} .
$$

To an $i$-site of the lattice, pertaining to a $\nu$-phase, one ascribes the variable $e_{i \nu}$ taking the value $e_{i \nu}=1$ when the site is occupied by a particle and the value $e_{i \nu}=0$ if the site is empty. The phase densities can be presented through the averages

$$
\rho_{\nu}=\frac{1}{V} \sum_{i=1}^{N_{s}}<e_{i \nu}>, \quad n_{\nu}=\frac{1}{N_{s}} \sum_{i=1}^{N_{s}}<e_{i \nu}>
$$

where $\langle\ldots\rangle$ implies a statistical averaging.

The procedure of averaging over heterophase fluctuations [2], for the considered case, yields the effective heterophase Hamiltonian

$$
\bar{H}=H_{1} \oplus H_{2}
$$

being a direct sum of the phase-replica Hamiltonians

$$
H_{\nu}=w_{\nu} \sum_{i=1}^{N_{s}}\left(K_{i}-\mu\right) e_{i \nu}+\frac{1}{2} w_{\nu}^{2} \sum_{i \neq j}^{N_{s}} \Phi_{i j} e_{i \nu} e_{j \nu},
$$

in which $K_{i}$ is kinetic energy, $\mu$ is chemical potential, and $\Phi_{i j}$ is interaction potential. The phase probabilities $w_{\nu}$ are defined by minimizing the grand potential

$$
\Omega=-\Theta \ln \operatorname{Tr} e^{-\beta \bar{H}} \quad(\beta \Theta \equiv 1)
$$

where $\Theta$ is average temperature in energy units. With the notation

$$
w \equiv w_{1}, \quad w_{2}=1-w,
$$

the minimization equations are

$$
\frac{\partial \Omega}{\partial w}=0, \quad \frac{\partial^{2} \Omega}{\partial w^{2}}>0 .
$$

The first of these equations leads to the equality

$$
<\frac{\partial \bar{H}}{\partial w}>=0
$$

The second of Eqs. (12) tells that the heterophase susceptibility

$$
\chi_{\Theta} \equiv \frac{1}{N_{s}} \frac{\partial^{2} \Omega}{\partial w^{2}}>0
$$

is to be positive. Differentiating the thermodynamic potential (10) gives

$$
\chi_{\Theta}=\frac{1}{N_{s}}\left[<\frac{\partial^{2} \bar{H}}{\partial w^{2}}>-\beta<\left(\frac{\partial \bar{H}}{\partial w}\right)^{2}>\right] .
$$


The inequality $\chi_{\Theta}>0$ is a condition of heterophase stability.

In order to illustrate in an explicit way the thermodynamic properties of the considered system, without plunging to mathematical complications, let us choose the long-range interaction potential $\Phi_{i j}=\Phi_{i j}\left(N_{s}\right)$ depending on the number of lattice sites so that

$$
\lim _{N_{s} \rightarrow \infty} \Phi_{i j}\left(N_{s}\right)=0, \quad \lim _{N_{s} \rightarrow \infty}\left|\frac{1}{N_{s}} \sum_{i \neq j}^{N_{s}} \Phi_{i j}\left(N_{s}\right)\right|<\infty .
$$

This type of interaction potentials allows one to get, in the thermodynamic limit, asymptotically exact solutions [31], which is due to the asymptotically exact decoupling

$$
<e_{i \nu} e_{j \nu}>=\delta_{i j}<e_{i \nu}>+\left(1-\delta_{i j}\right)<e_{i \nu}><e_{j \nu}>.
$$

For the compactness of the following expressions, let us introduce the notation

$$
K \equiv \frac{1}{N_{s}} \sum_{i=1}^{N_{s}} K_{i}, \quad \Phi \equiv \frac{1}{N_{s}} \sum_{i \neq j}^{N_{s}} \Phi_{i j},
$$

and let us define the dimensionless temperature and an effective chemical potential, respectively,

$$
T \equiv \frac{\Theta}{\Phi}, \quad \mu_{*} \equiv \frac{\mu-K}{\Phi} .
$$

Also, denote the dimensionless densities (5) as

$$
n_{1} \equiv a, \quad n_{2} \equiv b \quad(a>b) .
$$

Then for the grand potential (10), one obtains

$$
\begin{gathered}
\frac{\Omega}{\Phi N_{s}}=\frac{a}{2}(1-a) w^{2}+\frac{b}{2}(1-b)(1-w)^{2}-\frac{1}{2} \mu_{*}- \\
-T \ln \left[4 \cosh \left(\frac{a w^{2}-\mu_{*} w}{2 T}\right) \cosh \frac{b(1-w)^{2}-\mu_{*}(1-w)}{2 T}\right] .
\end{gathered}
$$

The heterophase susceptibility (15) can be reduced to the form

$$
\chi_{\Theta}=\left(n_{1}^{2}+n_{2}^{2}\right) \Phi=\left(a^{2}+b^{2}\right) \Phi .
$$

The condition of heterophase stability, $\chi_{\Theta}>0$, is nothing but the simple inequality

$$
\Phi>0 .
$$

Heterophase turbulence is possible for positive interaction strength $\Phi$, which plays here the role of the Reynolds number.

The condition of heterophase stability (23) is a necessary, but not sufficient, condition for the occurrence of heterophase turbulence. To show that the latter really develops, one has to check the existence of solutions for all thermodynamic characteristics. We have accomplished such an analysis by means of numerical calculations. Figure 1 demonstrates the temperature dependence of the partial densities (20) for different average densities n, and Fig. 2 presents 
the temperature dependence of the probability of the dense phase. Physically meaningful solutions require that the probability $w$ be in the interval $0 \leq w \leq 1$. Hence, heterophase turbulence can actually arise not for all densities and temperatures. The solutions for the partial densities (20), defined by the equations

$$
\begin{aligned}
& a=\frac{1}{2}-\frac{1}{2} \tanh \left[b(n-b) \frac{2 a b-n(a+b)}{2(a-b)^{3} T}\right], \\
& b=\frac{1}{2}-\frac{1}{2} \tanh \left[a(a-n) \frac{2 a b-n(a+b)}{2(a-b)^{3} T}\right],
\end{aligned}
$$

have sense only when the dense-phase probability

$$
w=\frac{n-b}{a-b} \quad(a>b)
$$

satisfies the inequalities $0 \leq w \leq 1$. The latter, together with the condition $a>b$, yield the requirements

$$
b<n<a, \quad 0<n<0.323 .
$$

Figures 3 and 4 show the effective chemical potential

$$
\mu_{*}=\frac{a^{2}(n-b)+b^{2}(n-a)}{(a-b)^{2}}
$$

and the dimensionless pressure

$$
P=-\frac{1}{\rho_{s} \Phi} \frac{\partial \Omega}{\partial V}
$$

as functions of temperature. The dimensionless specific heat

$$
C_{V}=-\frac{T}{N_{s} \Phi} \frac{\partial^{2} \Omega}{\partial T^{2}}
$$

and isothermal compressibility

$$
\kappa_{T}=-\frac{1}{V}\left(\frac{\partial P}{\partial V}\right)^{-1}
$$

are shown in Figs. 5 and 6, respectively. Their positiveness demonstrates the thermal and mechanical stability of the heterophase system. Finally, Fig. 7 demonstrates that the dimensionless grand potential $\omega_{w} \equiv \Omega / N_{s} \Phi$ for the heterophase matter is lower than that for the pure system, $\omega_{1}$, when $w=1$. This means that the state with heterophase turbulence is globally more stable than the state having no heterophase fluctuations.

In this way, there exists a region of thermodynamic variables, e.g. temperature $\Theta$ and density $\rho$, where the lattice system displaying heterophase turbulence is absolutely stable as a whole, despite that the emerging turbulence assumes local instability. That is, local instability renders the system more stable globally. 


\section{Origins of Heterophase Turbulence}

In general, there could be three causes producing heterophase turbulence, extrinsic, intrinsic, and one intermediate between the first two, which may be called stochastic [2].

The extrinsic cause presupposses the action of sufficiently strong external forces making the system nonequilibrium. These could be either specially designed time-dependent perturbations or uncontrolled random interactions with surrounding. This is similar to the way of preparing a turbulent fluid by strongly shaking the system [1], as a result of which the fluid becomes locally turbulent, i.e. nonuniform, but on the average it is uniform and isotropic, with zero average velocity. In the case of a solid, regions of disorder inside it could be formed by shock waves, irradiation by particle beams, electromagnetic fields etc. The possibility of producing heterophase turbulence by means of strong external forces looks rather evident, hence not as interesting. A more intriguing question is whether heterophase turbulence could emerge without strong external fields or not.

The intrinsic mechanism for the formation of heterophase fluctuations is based on the Boltzmann hypothesis [32] that in the entirely equilibrium system strongly nonequilibrium local fluctuations can appear in a spontaneous way. This mechanism of the appearance of nonequilibrium heterophase fluctuations from an equilibrium state was also advocated by Frenkel [33]. However, there is a slight inconsistence in this way of thinking. Really, first, one presupposses the existence of equilibrium states. Then one assumes that an equilibrium state can spontaneously pass to a nonequilibrium one. But the latter implies that equilibrium states are not absolutely stable, that is, they, actually, do not exist as stable states. Following this way of reasoning, it would be more logical to postulate from the very beginning that an absolutely stable state, to which an isolated system tends with time, is, generally, not an equilibrium state but a quasiequilibrium heterophase state [2].

The stochastic source generating heterophase turbulence is intermediate between the above two in the following sense. It accepts that completely isolated systems do not exist in reality, but any given system is always subject to uncontrollable random perturbations from the external surrounding. At the same time, one can prepare an almost isolated, or quasi-isolated, system for which external stochastic influence, if not completely eliminated, but is essentially reduced. Nevertheless, even infinitesimally small random perturbations can provoke noticeable chaoticity in a quasi-isolated system, with chaotic features governed by the system internal properties [2,34].

The fact that no real system can be ideally isolated has been repeatedly emphasized [35-39]. Moreover, it has been stressed [40,41] that the concept of an isolated system is self-contradictory by its own - To realize the isolation, one has to employ technical devices acting on the system, and to check that the latter is kept isolated, one needs to resort to measuring instruments perturbing the system.

In this section we show that even negligibly small stochastic perturbations can leed to the development of chaoticity in the system. The evolution equations are, generally, partial differential equations. Let a dynamical system be described by a set of $y(t)=\left[y_{i}(x, t)\right]$ of functions $y_{i}(x, t)$ enumerated by $i=1,2, \ldots, d$, with a continuous variable $x \in \mathbb{D}$ denoting a set of spatial variables pertaining to a domain $\mathbb{D}$, and $t \in \mathbb{R}_{+}$being time. The set $y(t)$, called the dynamical state, can be treated as a vector-column labelled by the discrete index $i$ and by the continuous multivariable $x$. The velocity field $v(y, t)=\left[v_{i}(x, y, t)\right]$ is also a column in the space of $i$ and $x$. Any system of evolution equations can be presented in the normal 
form

$$
\frac{d}{d t} y(t)=v(y, t)
$$

The velocity field $v(y, t)$ may contain differential and integral operations. The appearance of chaos is related to the problem of stability of evolution equations.

\subsection{Autonomous systems}

Before passing to more complicated cases, let us recall just in a few lines how the problem of stability is treated for autonomous systems of nonlinear partial differential equations. This introductory consideration is useful as far as in the standard textbooks one usually studies only the stability of nonlinear ordinary differential equations. At the same time this example will show how to deal with the matrix notation [34] that will be constantly used in what follows.

A dynamical system is autonomous if the velocity field does not depend explicitly on time, $v(y, t)=v(y)$. Then the system evolution is given by a set of equations

$$
\frac{d}{d t} y(t)=v(y)
$$

presented in the matrix form.

One looks for a stationary solutions $y^{*}$ defined by the zero velocity field,

$$
v\left(y^{*}\right)=0, \quad y^{*}=\left[y_{i}(x)\right] .
$$

For a small deviation

$$
\delta y^{*}(t)=y(t)-y^{*}
$$

from the stationary solution $y^{*}$, one gets a linearized equation

$$
\frac{d}{d t} \delta y^{*}(t)=\hat{J}^{*} \delta y^{*}(t)
$$

in which $\hat{J}^{*}=\left[J_{i j}^{*}\left(x, x^{\prime}\right)\right]$ is a contraction matrix with the elements

$$
J_{i j}^{*}\left(x, x^{\prime}\right) \equiv \frac{\delta v_{i}\left(x, y^{*}\right)}{\delta y_{j}^{*}\left(x^{\prime}\right)} .
$$

Looking for the solutions of the eigenproblem

$$
\hat{J}^{*} f_{n}=J_{n}^{*} f_{n}
$$

where $n$ is a multi-index, one finds the eigenvalues $J_{n}^{*}$ and the eigenfunctions $f_{n}=\left[f_{n i}(x)\right]$ of the contraction matrix. Assume that the eigenvalues form an orthonormalized and complete basis, $\left\{f_{n}\right\}$, so that

$$
f_{m}^{+} f_{n}=\delta_{m n}, \quad \sum_{n} f_{n} f_{n}^{+}=\hat{1} .
$$

Note that in the explicit presentation, the matrix eigenproblem (36) reads

$$
\sum_{j} \int J_{i j}^{*}\left(x, x^{\prime}\right) f_{n j}\left(x^{\prime}\right) d x^{\prime}=J_{n}^{*} f_{n i}(x) .
$$


Expanding the deviations

$$
\delta y^{*}(t)=\sum_{n} \delta c_{n}(t) f_{n}
$$

over the basis $\left\{f_{n}\right\}$, one has the equation

$$
\frac{d}{d t} \delta c_{n}(t)=J_{n}^{*} \delta c_{n}(t)
$$

for the coefficients $\delta c_{n}(t)$. This equation immediately gives

$$
\delta c_{n}(t)=\delta c_{n}\left(t_{0}\right) \exp \left\{J_{n}^{*}\left(t-t_{0}\right)\right\} .
$$

The stability of the stationary solutions is characterized by the Lyapunov exponents

$$
\lambda_{n} \equiv \lim _{t \rightarrow \infty} \frac{1}{t} \ln \left|\frac{\delta c_{n}(t)}{\delta c_{n}\left(t_{0}\right)}\right|=\operatorname{Re} J_{n}^{*} .
$$

The set $\left\{\lambda_{n}\right\}$ is the Lyapunov spectrum. The functions $f_{n}$ are termed normal modes. One tells that an $n$-mode is stable if $\lambda_{n}<0$, neutral when $\lambda_{n}=0$, and unstable for $\lambda_{n}>0$. The motion is chaotic if at least one mode is unstable.

\subsection{Stochastic systems}

Let us now turn to the problem of stability of stochastic differential equations. Assume that the system is subject to the action of stochastic fields $\xi(t)$. We continue using the matrix notation, where the dynamical state $y(\xi, t)=\left[y_{i}(x, \xi, t)\right]$ and the velocity field $v(y, \xi, t)=$ $\left[v_{i}(x, y, \xi, t)\right]$ are the columns in $i$ and $x$. The evolution of a stochastic dynamical system is given by the matrix equation

$$
\frac{d}{d t} y(\xi, t)=v(y, \xi, t) .
$$

Let the evolution equation (41) be complimented by the initial condition

$$
y(\xi, 0)=y(0),
$$

where $y(0)$ is a set of given functions $y_{i}(x)$. To solve a stochastic differential equation implies to find the solution

$$
y(t) \equiv \ll y(\xi, t) \gg
$$

averaged over the stochastic fields $\xi(t)$. We denote the stochastic averaging by the double angle brackets $\ll \ldots \gg$, reserving the single brackets $\langle\ldots\rangle$ for statistical averaging, as in Sec. 2.

Note that stochastic differential equations [42] can be defined either in the sense of Ito or in the sense of Stratonovich. We prefer to use the latter definition which involves simpler calculations, agreeing with the standard analysis, and which is better motivated physically [43]. Another possibility could be to present the stochastic fields $\xi(t)$ as expansions, with random coefficients over smooth functions of time [44,45]. This method makes it possible to employ the standard analysis in differentiating and integrating over time. The final results of the expansion approach $[44,45]$ coincide with those of the Stratonovich way. 
The stability of the stochastic equation (41) cannot be analysed in the same manner as the stability of the autonomous equation (31), since the stationary solutions for the stochastic equation (41) are not defined. Another approach is necessary. Let us introduce the local stability factor

$$
\nu(t) \equiv \sup _{\delta y(0)} \frac{|\delta y(t)|}{|\delta y(0)|}
$$

characterizing the maximal deviation of the average trajectory at time $t$ after an infinitesimal variation of initial conditions $\delta y(0) \rightarrow 0$. The following classification for the local property of motion is evident:

$$
\begin{array}{cc}
\nu(t)<1 & \text { locally stable } \\
\nu(t)=1 & \text { locally neutral } \\
\nu(t)>1 & \text { locally unstable }
\end{array}
$$

If the limit

$$
\nu(\infty)=\lim _{t \rightarrow \infty} \nu(t)
$$

exists, then one can tell that the motion is asymptotically stable for $\nu(\infty)<1$, asymptotically neutral for $\nu(\infty)=1$, and asymptotically unstable for $\nu(\infty)>1$. The quantity

$$
\lambda \equiv \lim _{t \rightarrow \infty} \frac{1}{t} \ln \nu(t)
$$

is the largest Lyapunov exponent.

The usage of the local stability factor (44) provides one with a much richer information on the motion than the largest Lyapunov exponent, since it is often important to study local in time stability but not only the asymptotic, as $t \rightarrow \infty$, property of motion [34]. Many dynamical systems display a very complicated structure of their phase space, containing different singular zones, such as trapping islands, because of which the fine local properties of orbits play a leading role, while fairly rough characteristics such as the Lyapunov exponent are less important [46]. In addition, for stochastic differential equations, the divergence of trajectories is not necessarily exponential [47]. For example, if $\nu(t) \sim t^{\beta}$, then the motion can be either asymptotically stable or unstable depending on whether $\beta<0$ or $\beta>0$, while the Lyapunov exponent $\lambda=0$ for both these cases characterizes the motion as neutral, thus, loosing information about the motion.

To calculate the stability factor $(44)$, we need to find $\delta y(t) \equiv \ll \delta y(\xi, t) \gg$. For this purpose, we introduce the stochastic multiplier matrix [34] as a matrix $\hat{M}(\xi, t)=\left[M_{i j}\left(x, x^{\prime}, \xi, t\right)\right]$ with the elements

$$
M_{i j}\left(x, x^{\prime}, \xi, t\right) \equiv \frac{\delta y_{i}(x, \xi, t)}{\delta y_{j}\left(x^{\prime}, 0\right)}
$$

and the contraction matrix $\hat{J}(\xi, t)=\left[J_{i j}\left(x, x^{\prime}, \xi, t\right)\right]$ with the elements

$$
J_{i j}\left(x, x^{\prime}, \xi, t\right) \equiv \frac{\delta v_{i}(x, y, \xi, t)}{\delta y_{j}\left(x^{\prime}, \xi, t\right)} .
$$

Accomplishing the variational differentiation of the evolution equation (41), we get the equation

$$
\frac{d}{d t} \hat{M}(\xi, t)=\hat{J}(\xi, t) \hat{M}(\xi, t)
$$


for the multiplier matrix. When for the latter the eigenproblem

$$
\hat{M}(\xi, t) \varphi_{n}(t)=\mu_{n}(\xi, t) \varphi_{n}(t)
$$

is defined, then the stability factor (44) becomes

$$
\nu(t)=\sup _{n}\left|\ll \mu_{n}(\xi, t) \gg\right| .
$$

The eigenvalues $\mu_{n}(\xi, t)$ of the stochastic multiplier matrix $\hat{M}(\xi, t)$ can be found from Eqs. (48) and (49).

\subsection{Quasi-isolated systems}

As is discussed in the beginning of this section, no real system can be ideally isolated, although the influence of random external perturbations could, probably, be made very small. Nevertheless, even arbitrarily small random perturbations could provoke system instability. To analyze this problem, we need to consider the stability of a given dynamical system not only with respect to the variation of initial conditions but also with respect to small stochastic perturbations.

Let the amplitude of stochastic fields be small, which can be presented by writing an explicit small factor $\alpha$ in front of $\xi(t)$. That is, instead of the evolution equation (36), we consider

$$
\frac{d}{d t} y(\alpha \xi, t)=v(y, \alpha \xi, t) \quad(\alpha \rightarrow 0),
$$

where the complex parameter $\alpha$ is infinitesimally small, so that $\alpha \rightarrow 0$ implies $\alpha_{1} \equiv \operatorname{Re} \alpha \rightarrow 0$ and $\alpha_{2} \equiv \operatorname{Im} \alpha \rightarrow 0$. When $\alpha \equiv 0$, no stochastic fields act on the system, and the evolution equation

$$
\frac{d}{d t} y(0, t)=v(y, 0, t)
$$

describes an isolated system. Switching on a small parameter models uncontrollable action of the surrounding. The parameter $\alpha$ is taken to be complex in order to simulate fluctuations of different physical quantities, for instance, of energy and attenuation, or of density and phase.

The stability of a stochastic system is characterized by the stability factor (50), which for Eq. (51) takes the form

$$
\nu(\varepsilon, t)=\sup _{n}\left|\ll \mu_{n}(\alpha \xi, t) \gg\right|,
$$

where $\varepsilon=\varepsilon\left(\alpha_{1}, \alpha_{2}\right)$ is a function of $\alpha_{1}=\operatorname{Re} \alpha$ and $\alpha_{2}=\operatorname{Im} \alpha$, such that $\varepsilon \rightarrow 0$ as $\alpha \rightarrow 0$. Depending on how $\alpha_{1} \rightarrow 0$ and $\alpha_{2} \rightarrow 0$, the parameter $\varepsilon$ can behave either as $\varepsilon \rightarrow-0$ or $\varepsilon \rightarrow+0$, or it can even be that $\varepsilon \equiv 0$. Of all these possibilities, one has to choose the case corresponding to the maximal stability factor (52), as far as the latter, by definition, characterizes the maximal possible divergence of initially close trajectories. Therefore, everywhere in what follows, the limit $\varepsilon \rightarrow 0$ is defined as

$$
\lim _{\varepsilon \rightarrow 0} \equiv \sup _{\varepsilon} \lim _{|\varepsilon| \rightarrow 0} .
$$


To analyze the asymptotic stability one has to look at the temporal limit $t \rightarrow \infty$. The result of taking the double limit of the stability factor (52) may depend on the order in which the limits $\varepsilon \rightarrow 0$ and $t \rightarrow \infty$ are taken. We shall say [34] that the system is stochastically stable if the order of these limits is not important, that is, the limits commute with each other,

$$
\left[\lim _{t \rightarrow \infty}, \lim _{\varepsilon \rightarrow 0}\right] \nu(\varepsilon, t)=0 .
$$

Conversely, the system is termed stochastically unstable, when these limits do not commute:

$$
\left[\lim _{t \rightarrow \infty}, \lim _{\varepsilon \rightarrow 0}\right] \nu(\varepsilon, t) \neq 0 .
$$

If a quasi-isolated system is stochastically unstable then, since any real system is never completely isolated but can be only quasi-isolated, chaos in the system can be induced by infinitesimally small random perturbations.

\section{Stochastic Density Matrices}

Now, employing the technique of the previous section, let us investigate if the evolution of particle density could be stochastically unstable. If so, this would mean that the density as a function of space and time could exhibit nonequilibrium fluctuations. Each of such fluctuations, after appearing, lives a finite lifetime, and then disappears. But, as time tends to infinity, the density does not tend to an equilibrium, say uniform or strictly periodic in space, state. As far as the system is stochastically unstable, nonequilibrium fluctuations arise again and again, chaotically emerging in time as well as in space. The state of matter with such chaotic fluctuations would be representing heterophase turbulence.

The density of particles

$$
\rho(\mathbf{r}, t) \equiv \lim _{\mathbf{r}^{\prime} \rightarrow \mathbf{r}} \rho_{1}\left(\mathbf{r}, \mathbf{r}^{\prime}, t\right)
$$

as a function of the Cartesian vector $\mathbf{r}$ and time $t$, is a diagonal element of the single density matrix

$$
\rho_{1}\left(\mathbf{r}, \mathbf{r}^{\prime}, t\right) \equiv<\psi^{\dagger}\left(\mathbf{r}^{\prime}, t\right) \psi(\mathbf{r}, t)>
$$

in which the brackets $\langle\ldots\rangle$ imply statistical averaging and $\psi(\mathbf{r}, t)$ is a field operator, being a vector-column in the space of internal degrees of freedom, such as spin, isospin, and so on. The evolution of the single density matrix (57) is coupled with that of the double density matrix

$$
\rho_{2}\left(\mathbf{r}_{1}, \mathbf{r}_{2}, \mathbf{r}_{1}^{\prime}, \mathbf{r}_{2}^{\prime}, t\right) \equiv<\psi^{\dagger}\left(\mathbf{r}_{2}^{\prime}, t\right) \psi^{\dagger}\left(\mathbf{r}_{1}^{\prime}, t\right) \psi\left(\mathbf{r}_{1}, t\right) \psi\left(\mathbf{r}_{2}, t\right)>
$$

General properties of density matrices can be found in the book [48] (for some recent developments see Refs. [49,50]).

The evolution equation for the density matrix (57) can be presented [39] in the form

$$
\begin{gathered}
i \frac{\partial}{\partial t} \rho_{1}\left(\mathbf{r}_{1}, \mathbf{r}_{2}, t\right)=\left[-\frac{1}{2 m_{0}}\left(\nabla_{1}^{2}-\nabla_{2}^{2}\right)+U\left(\mathbf{r}_{1}, t\right)-U\left(\mathbf{r}_{2}, t\right)\right] \rho_{1}\left(\mathbf{r}_{1}, \mathbf{r}_{2}, t\right)+ \\
+\int\left[\Phi\left(\mathbf{r}_{1}, \mathbf{r}_{3}\right)-\Phi\left(\mathbf{r}_{2}, \mathbf{r}_{3}\right)\right] \rho_{2}\left(\mathbf{r}_{3}, \mathbf{r}_{1}, \mathbf{r}_{3}, \mathbf{r}_{2}, t\right) d \mathbf{r}_{3}
\end{gathered}
$$

where we set the Planck constant $\hbar \equiv 1, m_{0}$ is particle mass, and $U(\mathbf{r}, t)$ is an external potential, while $\Phi\left(\mathbf{r}, \mathbf{r}^{\prime}\right)=\Phi\left(\mathbf{r}^{\prime}, \mathbf{r}\right)$ is an interaction potential. 
When $\mathbf{r}_{2} \rightarrow \mathbf{r}_{1}$, then Eq. (59) reduces to the asymptotic form

$$
\frac{\partial}{\partial t} \rho_{1}\left(\mathbf{r}_{1}, \mathbf{r}_{2}, t\right)=\frac{i}{2 m_{0}}\left(\nabla_{1}^{2}-\nabla_{2}^{2}\right) \rho_{1}\left(\mathbf{r}_{1}, \mathbf{r}_{2}, t\right)
$$

Taking account of the equality

$$
\nabla_{1}^{2}-\nabla_{2}^{2}=\left(\nabla_{1}+\nabla_{2}\right)\left(\nabla_{1}-\nabla_{2}\right)
$$

and introducing [39] the density-of-current matrix

$$
\mathbf{j}\left(\mathbf{r}_{1}, \mathbf{r}_{2}, t\right) \equiv-\frac{i}{2 m_{0}}\left(\nabla_{1}-\nabla_{2}\right) \rho_{1}\left(\mathbf{r}_{1}, \mathbf{r}_{2}, t\right)
$$

one can rewrite Eq. (60) as

$$
\frac{\partial}{\partial t} \rho_{1}\left(\mathbf{r}_{1}, \mathbf{r}_{2}, t\right)=-\left(\nabla_{1}+\nabla_{2}\right) \mathbf{j}\left(\mathbf{r}_{1}, \mathbf{r}_{2}, t\right)
$$

The density of current is the diagonal element

$$
\mathbf{j}(\mathbf{r}, t) \equiv \lim _{\mathbf{r}^{\prime} \rightarrow \mathbf{r}} \mathbf{j}\left(\mathbf{r}, \mathbf{r}^{\prime}, t\right)
$$

which can also be represented as the statistical average

$$
\mathbf{j}(\mathbf{r}, t)=<\hat{\mathbf{j}}(\mathbf{r}, t)>
$$

of the density-of-current operator

$$
\hat{\mathbf{j}}(\mathbf{r}, t) \equiv-\frac{i}{2 m_{0}}\left[\psi^{\dagger} \nabla \psi-\left(\nabla \psi^{\dagger}\right) \psi\right]
$$

where, for brevity, we write $\psi \equiv \psi(\mathbf{r}, t)$. In the limit $\mathbf{r}_{2}=\mathbf{r}_{1}$, Eq. (62) transforms to the standard continuity equation

$$
\frac{\partial}{\partial t} \rho_{1}(\mathbf{r}, t)-\nabla \cdot \mathbf{j}(\mathbf{r}, t)=0
$$

Hence, we may say that Eq. (60) asymptotically, as $\mathbf{r}_{2} \rightarrow \mathbf{r}_{1}$, equivalent to the continuity equaiton (65) defining the evolution of the particle density (56).

To check the stochastic stability of the evolution equation (60), one has to incorporate there a small stochastic term. For the compactness of notation, let us denote $x=\left\{\mathbf{r}_{1}, \mathbf{r}_{2}\right\}$. Then an asymptotically small stochastic field is $\alpha \xi(x, t)$, with $\alpha \rightarrow 0$. Also define

$$
y(x, \alpha \xi, t) \equiv \rho_{1}\left(\mathbf{r}_{1}, \mathbf{r}_{2}, \alpha \xi, t\right) .
$$

The stochastic equation, obtained from the evolution equation (60), reads

$$
\frac{\partial}{\partial t} y(x, \alpha \xi, t)=v(x, y, \alpha \xi, t)
$$

where the velocity field is

$$
v(x, y, \alpha \xi, t)=\left[\frac{i}{2 m_{0}}\left(\nabla_{1}^{2}-\nabla_{2}^{2}\right)+\alpha \xi(x, t)\right] y(x, \alpha \xi, t) .
$$


The stochastic term initiates here fluctuations of energy and of attenuation leading to the related fluctuations of the amplitude and phase of the density matrix.

Following the strategy of the previous section, we find the contraction matrix (47),

$$
J\left(x, x^{\prime}, \alpha \xi, t\right)=\left[\frac{i}{2 m_{0}}\left(\nabla_{1}^{2}-\nabla_{2}^{2}\right)+\alpha \xi(x, t)\right] \delta\left(x-x^{\prime}\right) .
$$

Since $\alpha \rightarrow 0$, the eigenvalues of the matrix (69) can be found by means of perturbation theory. In the zero approximation, the normalized eigenfunctions of the contraction matrix are

$$
\varphi_{k}(x)=\frac{1}{V} \exp \left\{i\left(\mathbf{k}_{1} \cdot \mathbf{r}_{1}+\mathbf{k}_{2} \cdot \mathbf{r}_{2}\right)\right\}
$$

with the notation $k=\left\{\mathbf{k}_{1}, \mathbf{k}_{2}\right\}$. The eigenvalues of the contraction matrix, in the first order, are

$$
J_{k}(\alpha \xi, t)=\int \varphi_{k}^{*}(x) J\left(x, x^{\prime}, \alpha \xi, t\right) \varphi_{k}\left(x^{\prime}\right) d x d x^{\prime} .
$$

This, with the functions (70), gives

$$
J_{k}(\alpha \xi, t)=-\frac{i}{2 m_{0}}\left(\mathbf{k}_{1}^{2}-\mathbf{k}_{2}^{2}\right)+\alpha \xi_{k}(t)
$$

where

$$
\xi_{k}(t) \equiv \int \varphi_{k}^{*}(x) \xi(x, t) \varphi_{k}(x) d x
$$

Note that if $\xi(x, t)=\xi(t)$ is a purely temporal perturbation, then Eqs. (70) and (72) give exact eigenfunctions and eigenvalues of the contraction matrix.

The eigenfunctions (70) are stationary, which means [34] that in this approximation the multiplier matrix (46) possesses the same eigenfunctions. The eigenvalues of the multiplier matrix (46) are given by the eigenproblem

$$
\int M\left(x, x^{\prime}, \alpha \xi, t\right) \varphi_{k}\left(x^{\prime}\right) d x^{\prime}=\mu_{k}(\alpha \xi, t) \varphi_{k}(x) .
$$

Taking into account the equation (48) for the multiplier matrix, one has

$$
\mu_{k}(\alpha \xi, t)=\exp \left\{-\frac{i}{2 m_{0}}\left(k_{1}^{2}-k_{2}^{2}\right) t+\alpha \int_{0}^{t} \xi_{k}\left(t^{\prime}\right) d t^{\prime}\right\}
$$

Assuming that $\xi(x, t)$ is a real Gaussian stochastic variable, centered at zero, for the stability factor (52), having here the form

$$
\nu(\varepsilon, t)=\sup _{k}\left|\ll \mu_{k}(\alpha \xi, t) \gg\right|
$$

we obtain

$$
\nu(\varepsilon, t)=\sup _{k} \exp \left\{\varepsilon \int_{0}^{t} \ll \xi_{k}\left(t^{\prime}\right) \xi_{k}\left(t^{\prime \prime}\right) \gg d t^{\prime} d t^{\prime \prime}\right\},
$$

with $\varepsilon \equiv \frac{1}{2}\left(\alpha_{1}^{2}-\alpha_{2}^{2}\right)$. Keeping in mind that $\varepsilon \rightarrow 0$ according to the limit (53), the factor (76) can be rewritten as

$$
\nu(\varepsilon, t)=\exp \{|\varepsilon| \Gamma(t) t\}
$$


where

$$
\Gamma(t) \equiv \sup _{k} \frac{1}{t}\left|\int_{0}^{t} \ll \xi_{k}\left(t^{\prime}\right) \xi_{k}\left(t^{\prime \prime}\right) \gg d t^{\prime} d t^{\prime \prime}\right|
$$

It is evident that

$$
\lim _{t \rightarrow \infty} \lim _{\varepsilon \rightarrow 0} \nu(\varepsilon, t)=1 .
$$

However, under fixed $|\varepsilon|>0$, it may happen that the limit of $\nu(\varepsilon, t)$ as $t \rightarrow \infty$, does not exist, which could occur e.g. if $\Gamma(t)$ oscillates. Or it may be that $\Gamma(t) t \rightarrow \infty$, as $t \rightarrow \infty$, which results in $\nu(\varepsilon, t) \rightarrow \infty$. The latter behaviour can be illustrated by assuming that $\xi(x, t)$ describes the temporal white noise with the correlation function

$$
\ll \xi(x, t) \xi\left(x^{\prime}, t^{\prime}\right) \gg=\gamma \delta\left(t-t^{\prime}\right)
$$

where $\gamma>0$. Then $\Gamma(t)=\gamma$, and the factor (77) becomes

$$
\nu(\varepsilon, t)=\exp (|\varepsilon| \gamma t)
$$

Another possibility could be to consider the uniform noise with the correlator

$$
\ll \xi(x, t) \xi\left(x^{\prime}, t^{\prime}\right) \gg=\gamma^{2}
$$

Then $\Gamma(t)=\gamma^{2} t$, and the factor $(77)$ reduces to

$$
\nu(\varepsilon, t)=\exp \left(|\varepsilon| \gamma^{2} t^{2}\right)
$$

In any of such cases,

$$
\lim _{\varepsilon \rightarrow 0} \lim _{t \rightarrow \infty} \nu(\varepsilon, t)=\infty,
$$

which demonstrates stochastic instability of the system, in agreement with definition (55).

\section{Conclusion}

The considered simple cases show that there always exist such infinitessimaly small random perturbations which make the system stochastically unstable. In general, for stochastic instability, it is sufficient that

$$
\lim _{t \rightarrow \infty} \Gamma(t) t=\infty
$$

There exists a wide class of noises, for instance, ranging between the white noise and the uniform noise, which could provide the validity of this limit.

Instability of an evolution equation implies that there are no stationary solutions for this equation. A chaotic dynamical system can only possess a chaotic attractor. The above arguments show that the evolution of the particle density (56) can be stochastically unstable. Hence, the density cannot tend, with time, to a stationary limit describing a uniform density for liquids or gases and a periodic density for crystals. But the density $\rho(\mathbf{r}, t)$ will always depend on time, occasionally displaying random spatio-temporal fluctuations of nonequilibrium nature. To be noticeable as nonequilibrium, these fluctuations have to be at least mesoscopic. Such nonequilibrium mesoscopic fluctuations make the system heterophase. The state of matter with heterophase fluctuations represents heterophase turbulence. 
In this sense, there should exist turbulent crystals, whose density displays chaotic spatiotemporal fluctuations corresponding to mesoscopic regions of disorder. A turbulent crystal, being observed during the times shorter or of the order of the characteristic lifetime of heterophase fluctuations, $\tau_{c o h}$, would be seen as a matter, in which the spatial regions having approximately a crystalline structure with a type of lattice symmetry are randomly intermixed with disordered regions having no symmetry. The turbulent crystal, although being turbulent, is anyway a crystal because, being observed during the times much longer than the heterophase coherence time $\tau_{c o h}$, possesses on the average a periodic density, with a well-defined symmetry of crystalline lattice. The corresponding density, averaged over such long times $t \gg \tau_{c o h}$, can be written as

$$
\bar{\rho}(\mathbf{r}) \equiv \lim _{t \rightarrow \infty} \frac{1}{t} \int_{0}^{t} \rho\left(\mathbf{r}, t^{\prime}\right) d t^{\prime}
$$

Assuming heterophase quasiergodicity [2], the average density writes

$$
\bar{\rho}(\mathbf{r})=w_{1} \rho_{1}(\mathbf{r})+w_{2} \rho_{2}
$$

where $w_{1}$ and $w_{2}$ are statistical weights of the crystalline phase and of the disordered phase, respectively. The crystalline density $\rho_{1}(\mathbf{r})$ is periodic, while that of the disordered phase is uniform. For the densities averaged over space,

$$
\rho \equiv \int \bar{\rho}(\mathbf{r}) d \mathbf{r}, \quad \rho_{\nu} \equiv \int \rho_{\nu}(\mathbf{r}) d \mathbf{r},
$$

we return to the equality (4).

The possibility for the existence of turbulent crystals does not require that all crystals be necessarily turbulent. Whether they are such or not depends on the internal properties of the system as well as on external conditions, as is demonstrated in the model picture of section 2. But this possibility to become turbulent must be taken into account. Heterophase turbulence may be absent at some particular conditions, but may emerge when changing the latter. For example, it may not exist at low temperatures, but can arise near the temperature of melting $[16,17]$, where the very existence of the heterophase turbulence is necessary for understanding the physics of melting and crystallization. 


\section{References}

[1] L.D. Landau and E.M. Lifshitz, Hydrodynamics (Nauka, Moscow, 1986).

[2] V.I. Yukalov, Phys. Rep. 28, 395 (1991).

[3] L.D. Faddeev, Ed., Mathematical Physics, (Grand Russian Encyclopedia, Moscow, 1998)

[4] D. Ruelle, Physica D7, 40 (1983).

[5] Y.A. Alexandrov, Fundamental Properties of Neutron (Clarendon, Oxford, 1992).

[6] D.P. Kharakoz and E.A. Shlyapnikova, J. Phys. Cem. B104, 10368 (2000).

[7] V.I. Yukalov, Int. J. Mod. Phys. B6, 91 (1992).

[8] G. Benedek and K.A. Müller, Eds., Phase Separation in Cuprate Superconductors (World Scientific, Singapore, 1992).

[9] E. Sigmund and K.A. Müller, Eds., Phase Separation in Cuprate Superconductors, (Springer, Berlin, 1994).

[10] A.J. Coleman, E.P. Yukalova, and V.I. Yukalov, Physica C243, 76 (1995).

[11] Y.L. Khait, Atomic Diffusion in Solids (Scitec, Zurich, 1997).

[12] B. Sundaram and G.M. Zaslavsky, Phys. Rev. E59, 7231 (1999).

[13] R.D. Merithew et. al., Phys. Rev. Lett. 84, 3442 (2000).

[14] R. Caciuffo et al., Phys. Rev. B59, 13892 (1999).

[15] D. Mannix et al., Phys. Rev. B60, 15187 (1999).

[16] V.I. Yukalov, Phys. Lett. A81, 433 (1981).

[17] V.I. Yukalov, Phys. Rev. B32, 436 (1985).

[18] H. Tanaka, J. Phys. Condens. Matter 10, L207 (1998).

[19] H. Tanaka, J. Phys. Condens. Matter 11, L159 (1999).

[20] H. Tanaka, Europhys. Lett. 50, 340 (2000).

[21] D. Sornette, Phys. Rep. 313, 237 (1999).

[22] G. Neergaard and J. Madsen, Preprint hep-ph/9807272 (1999).

[23] G. Neergaard and J. Madsen, Preprint hep-ph/0003176 (2000).

[24] J.S. Smart, Effective Field Theories in Magnetism (Saunders, Philadelphia, 1966).

[25] A. Ziegler, J. Mag. Mag. Mat. 104, 669 (1992). 
[26] D.I. Pushkarov, Quasiparticle Theory of Defects in Solids (World Scientific, Singapore, 1991).

[27] V.I. Yukalov and V.I. Zubov, Fortschr. Phys. 31, 627 (1983).

[28] V.I. Zubov, C.J. Rodrigues, and M.F. Pascual, Int. J. Mod. Phys. B12, 2869 (1998).

[29] V.I. Yukalov and E.P. Yukalova, Physica A213, 482 (1995).

[30] V.I. Yukalov, Int. J. Mod. Phys. B3, 311 (1989).

[31] N.N. Bogolubov, Method for Studying Model Hamiltonians (Pergamon, Oxford, 1972).

[32] L. Boltzmann, Vorlesungen über Gastheorie (Barth, Leipzig, 1896).

[33] J.I. Frenkel, Statistical Physics (Academy of Sciences, Moscow, 1948).

[34] V.I. Yukalov, Physica A234, 725 (1997).

[35] D. Ter Haar, Elements of Statistical Mechanics (Rinehart, New York, 1954).

[36] L.D. Landau and E.M. Lifshitz, Statistical Mechanics (Pergamon, Oxford, 1958).

[37] J.E. Mayer and M.G. Mayer, Statistical Mechanics (Wiley, New York, 1977).

[38] O. Penrose, Rep. Prog. Phys. 42, 1937 (1979).

[39] V.I. Yukalov, Statistical Green's Functions (Queen's University, Kingston, 1998).

[40] V.I. Yukalov, Moscow Univ. Phys. Bull. 25, 49 (1970).

[41] V.I. Yukalov, Moscow Univ. Phys. Bull. 26, 22 (1971).

[42] C.W. Gardiner, Handbook of Stochastic Methods (Springer, Berlin, 1985).

[43] R.L. Stratonovich, Nonlinear Nonequilibrium Thermodynamics (Moscow, Nauka, 1985).

[44] V.I. Yukalov, Phys. Rev. A56, 5004 (1997).

[45] V.I. Yukalov, Laser Phys. 7, 998 (1997).

[46] G.M. Zaslavsky, M. Edelman, and B.A. Niyazov, Chaos 7, 159 (1997).

[47] X. Mao, Stability of Stochastic Differential Equations with Respect to Semimartingales (Longman, Harlow, 1991).

[48] A.J. Coleman and V.I. Yukalov, Reduced Density Matrices (Springer, Berlin, 2000).

[49] D.A. Mazziotti, J. Chem. Phys. 112, 10125 (2000).

[50] D.A. Mazziotti and D.R. Herschbach, Phys. Rev. A62, 043603 (2000). 


\section{Figure Captions}

Fig. 1. The partial densities of the dense phase (solid curve) and of the dilute phase (dashed curve), for different average densities $n$, as functions of temperature.

Fig. 2. The probability of the dense phase versus temperature for different $n$.

Fig. 3. The effective chemical potential vs. temperature.

Fig. 4. The dimensionless pressure as a function of temperature.

Fig. 5. The dimensionless specific heat vs. temperature.

Fig. 6. The isothermal compressibility vs. temperature.

Fig. 7. The dimensionless grand potential for the heterophase matter, $\omega_{w}$, and for the pure dense system, $\omega_{1}$, vs. temperature. 